\title{
5 \\ Stable Testers for Environment Sensitive Systems
}

\author{
Mohammed Ghriga \\ Department of Computer Science, Long Island University, \\ 1 University Plaza, Brooklyn, NY 11201 USA \\ E-mail: mghriga@comsci.liunet.edu
}

\begin{abstract}
We present a new testing technique for a restricted class of nondeterministic systems, we qualify as environment sensitive. Such systems are specified and implemented such that all nondeterministic transitions, whether internal or not, are conditionally driven function of a system's environment conditions. The rationale and the practical aspects of this class will be given. The intention is to provide a pragmatic framework to improve the testability aspects of nondeterministic systems. We show that our restricted class of systems coupled with a new testing postulate Wait and Lock Stability lead to testing conditions similar to those of traditional sequential/deterministic systems; the testing postulate is analyzed and an algorithmic characterization for the construction of testers, that are both sequential and stable, from system specifications provided. Finally, we establish the correlation of our results and testing approach to the related work in failure and failure trace semantics when applicable.
\end{abstract}

Keywords

Conformance Testing, Labelled Transition Systems, Testing with Deadlock Detection.

\section{Introduction}

Conformance testing is known to be a problem of considerable complexity. Nevertheless, it is crucial in that it helps ensures interoperability among multi-vendor products. Significant efforts have been expended to establish testing theories and frameworks, formulate methodologies for the generation of conformance tests, and develop techniques to optimize such tests. Yet, many problems and issues remain open as indicated by Cavalli, Favreau and Phalippou (1994). This paper attempts to: (1) investigate notions of implementation in conjunction with a restricted class of nondeterministic systems, we will qualify as environment sensitive; and (2) link test execution procedures to current practices in testing 
sequential/deterministic systems. We use notion of implementation to simply refer to the conditions under which an implementation is said to satisfy its specification.

Communicating systems can be described as processes whose dynamic behaviors are formalized as labelled transition systems (LTS). A system is said to be environment sensitive if all nondeterministic transitions in the system, whether internal or not, are conditionally driven by current environment conditions rather than being implicitly driven. Such environment sensitive systems can be of particular interest to industry in various domains, e.g.: client-server applications, communicating systems, critical-safety systems, etc. These issues will be made clearer in Section 2.

Testing LTS-based implementations has concentrated on notions of implementation based on trace semantics (exemplified by Cavalli, Kim and Maigron (1993)), failure semantics (exemplified by Brinksma (1988); Brinksma, Scollo and Steenbergen (1987); Tretmans (1994); Drira (1994); Fujiwara and Bochman (1992); Pitt and Freestone (1990)), and failure trace semantics (Langerak, 1990). The most detailed work on failure semantics is due to Brinksma (1988). Unfortunately, the implied notions of implementation may not be satisfactory as indicated by Langerak (1990). This same criticism of inadequate discrimination applies with respect to the class of environment sensitive systems. On the other hand, we argue that Langerak's notions of implementation are too expensive to be of practical interest. This paper proposes a more practical and pragmatic notion of testers that preserve the intuitive behavior induced by nondeterminism.

The paper is organized as follows. In Section 2, we formally define the class of environment sensitive systems and project on their practical aspects. In section 3 , we present the elements of our testing approach and the concept of stability locks as well as the feasibility of testing. In Section 4, we give a characterization of finite LTSs. In Section 5, we propose an algorithmic treatment of internal events based on a simple fragmentation process. In section 6 , we provide an algorithmic characterization of our testers and define their induced notion of implementation. In Section 7, we examine/establish the relationships between our work and the related work in both failure and failure trace semantics. Section 8 concludes the paper.

\section{Environment Sensitive Systems}

In this section, we formally define the notion of environment sensitive systems, illustrate their basic concepts, and discuss their practical aspects.

We first give the definition of a labelled transition system (LTS). An LTS $S$ is a 4-tuple $S=\left(Q, \sum, \Delta, s_{0}\right)$, where $Q$ is a countable non-empty set of states, $\sum$ a countable set of observable actions, $\Delta$ a set of transitions which is a subset of $Q \times\left(\sum \cup\{\tau\}\right) \times Q$ with $\tau \notin \sum$ being the unobservable or internal action, and $s_{0} \in Q$ the initial state of $S$.

An element $\left(s, \mu, s^{\prime}\right) \in \Delta$ is interchangeably written as $s \stackrel{\mu}{\longrightarrow} s^{\prime}$. The main notational conventions are given in Figure 1, where the $\mu_{i}$ s and $\mu$ are in $\sum \cup\{\tau\}$ and $S$ is an LTS with $s_{0}$ as an initial state. A state $s$ is said to be stable if $\neg(s \stackrel{\tau}{\longrightarrow})$ (that is, there is no outgoing transition with label $\tau$ at $s$ ), and unstable otherwise (Petrenko, Bochmann and Dssouli, 1994). An LTS is stable if all its states are stable, and unstable otherwise. 


\begin{tabular}{|c|c|}
\hline Notation & Meaning \\
\hline $\begin{array}{l}\stackrel{\mu_{1} \mu_{2} \ldots \mu_{n}}{\Longrightarrow} s^{\prime} \\
s \stackrel{\epsilon}{\Longrightarrow} s^{\prime} \\
s \stackrel{\mu}{\Longrightarrow} s^{\prime} \\
\stackrel{\mu_{1} \mu_{2} \ldots \mu_{n}}{\rightleftharpoons} s^{\prime} \\
s \stackrel{\mu_{1} \stackrel{\mu_{2} \ldots \mu_{n}}{\Longrightarrow}}{\Longrightarrow}\end{array}$ & $\begin{array}{l}\exists s_{i}(1 \leq i \leq n) s \stackrel{\mu_{1}}{\longrightarrow} s_{1} \stackrel{\mu_{2}}{\longrightarrow} \ldots \stackrel{\mu_{n}}{\longrightarrow} s_{n}=s^{\prime} \\
s \equiv s^{\prime} \text { or } \exists n \geq 1 s \stackrel{\tau^{n}}{\longrightarrow} s^{\prime} \\
\exists s_{1}, s_{2} s \stackrel{c}{\Longrightarrow} s_{1} \stackrel{\mu}{\longrightarrow} s_{2} \stackrel{c}{\Longrightarrow} s^{\prime} \\
\exists s_{i}(1 \leq i \leq n) s=s_{0} \stackrel{\mu_{1}}{\longrightarrow} s_{1} \stackrel{\mu_{2}}{\longrightarrow} \ldots \stackrel{\mu_{n}}{\longrightarrow} s_{n}=s^{\prime} \\
\exists s^{\prime} s \stackrel{\mu_{1} \mu_{2} \ldots \mu_{n}}{\longrightarrow} s^{\prime}\end{array}$ \\
\hline $\begin{array}{l}s \stackrel{\mu_{1} \mu_{2} \ldots \mu_{n}}{\Rightarrow} \Rightarrow \\
\operatorname{out}(s) \\
\operatorname{Tr}(s) \\
\operatorname{Tr}(S)\end{array}$ & $\begin{array}{l}\neg \exists s^{\prime} s^{\mu_{1} \mu_{2} \ldots \mu_{n}} s^{\prime} \\
=\left\{\mu \in \sum \mid s \stackrel{\mu}{\Longrightarrow}\right\} \\
=\left\{\sigma \in \sum^{*} \mid s \stackrel{\sigma}{\Longrightarrow}\right\} \\
=\operatorname{Tr}\left(s_{0}\right)\end{array}$ \\
\hline $\begin{array}{l}s \text { ref } A \\
S \text { after } \sigma \\
(S \text { after } \sigma) \text { ref } A \\
N_{\tau}(S)\end{array}$ & $\begin{array}{l}\forall \mu \in A: s \stackrel{\mu}{\Longrightarrow} \text { (where } A \subseteq \sum \text { ) } \\
\left.=\left\{s \mid s_{0} \stackrel{\sigma}{\Longrightarrow} s\right\} \text { (where } \sigma \in \Sigma^{*}\right) \\
\exists s \in(S \text { after } \sigma): s \text { ref } A \\
\text { number of } \tau \text { labelled transitions in } S\end{array}$ \\
\hline
\end{tabular}

Figure 1: Notations for Labelled Transition Systems.

Definition 1 An environment sensitive system $E$ is a 3-tuple $E=<S^{E}$,env $v_{p}^{E}$,env $v_{i p}^{E}>$, where $S^{E}$ is an LTS specification of $E$, env $v_{p}^{E}$ a countable set of (measurable) environment parameters of $E$, and env $v_{i p}^{E}$ a finite (ordered) list of predicates that are boolean functions of the environment parameters of $E$.

Let $E$ be an environment sensitive system (ESS). The set $e n v_{p}^{E}$ contains basically the parameters of $E$ 's environment that can influence the dynamic behavior of $E$. These parameters are used as variables in the predicates of $e n v_{i p}^{E}$ that are used to describe the interaction policies of $E$. Thus, we use the term interaction policy condition instance (IPCI) to refer to a sequence of $0 \mathrm{~s}$ and $1 \mathrm{~s}$, where the $i^{\text {th }}$ value (either 0 or 1 ) in the sequence is the value of the $i^{\text {th }}$ predicate in the list $e n v_{i p}^{E}$. It is assumed that all nondeterministic transitions in $S^{E}$, whether internal or not, are conditionally driven by the predicates in $e n v_{i p}^{E} . E$ is said to be stable iff $S^{E}$ is stable. Throughout this paper, we assume a tree representation of $S^{E}$. These trees, which are generally infinite, will be called tree labelled transition systems (TLTS). We let $T L T S\left(S^{E}\right)$ denote the tree representation of $S^{E}$. We assume that these processes do not contain divergences (i.e. no infinite chains of internal actions/transitions). Moreover, we assume that $\operatorname{Event}\left(s \longrightarrow s^{\prime}\right)$ returns the label of the edge $s \longrightarrow s^{\prime}$ and parent $(s)$ returns the parent of node $s$ in the tree representation. Note parent $\left(s_{0}\right)$ is nil.

Inspired by Langerak (1990), we give two simple examples from the realm of coffee/tea vending machines. We view tea ( $t)$ and coffee $(c)$ as measurable resources in these machines. Let $A_{t}$ (for tea) and $A_{c}$ (for coffee) be parameters that indicate the availability of such resources. 


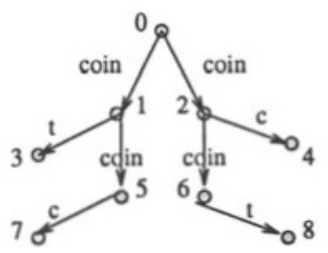

(a)

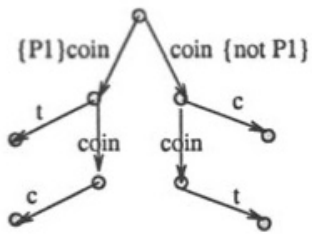

(b)

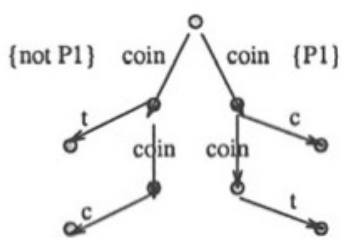

(c)

Figure 2: LTS Specification of $E_{1}$ and its Interpretations with $P_{1}=A_{t} \geq A_{c}$. (a) Specification $S^{E_{1}}$. (b) An Interpretation of $E_{1}$. (c) Another Possible Interpretation of $E_{1}$.

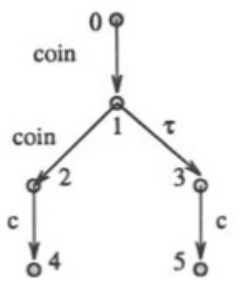

(a)

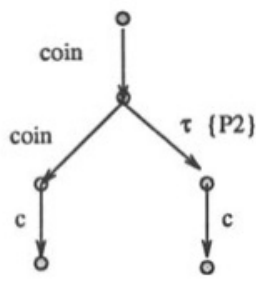

(b)

Figure 3: LTS Specification of $E_{2}$ and its Interpretation with $P_{2}=A_{c} \geq \kappa$. (a) Specification $S^{E_{2}}$ of $E_{2}$. (b) Interpretation of $E_{2}$.

Example 1 Let $E_{1}=<S^{E_{1}}$,env $v_{p}^{E_{1}}$,env $v_{i p}^{E_{1}}>$, where $S^{E_{1}}$ is depicted in Figure 2, env $v_{p}^{E_{1}}=$ $\left\{A_{t}, A_{c}\right\}$, and env $v_{i p}^{E_{1}}=\left[P\left(A_{t}, A_{c}\right), \neg P\left(A_{t}, A_{c}\right)\right]$ with $P\left(A_{t}, A_{c}\right)=A_{t} \geq A_{c}$.

As in (Langerak, 1990), $E_{1}$ always gives us what we want (Figure 2(a)). However, the cost for a desired choice (i.e. drink) depends on the availability of resources (i.e. tea and coffee). The possible IPCIs of $E_{1}$ are the sequences [01] and [10]; the other possible sequences are infeasible given $e n v_{i p}^{E_{1}}$. Figure 2(b), (c) give the possible interpretations of $E_{1}$, which differ in the attachment of the predicates to the nondeterministic transitions. In Figure 2(b) and under IPCI [10] (for example), if one desires coffee, he/she has to insert an additional coin to get coffee after the refusal of the first trial for getting coffee. Similarly, other scenarios (that may or may not contain refusals) can be derived.

Example 2 Let $E_{2}=<S^{E_{2}}$,env $v_{p}^{E_{2}}$,env $v_{i p}^{E_{2}}>$, where $S^{E_{2}}$ is depicted in Figure 3, env $v_{p}^{E_{2}}=$ $\left\{A_{c}\right\}$, and env $v_{i p}^{E_{1}}=\left[P\left(A_{c}\right)\right]$ with $P\left(A_{c}\right)=A_{c} \geq \kappa(\kappa$ is a constant).

Again, $E_{2}$ always give us coffee, but the cost is always enforced to be two coins if the availability of coffee is below certain threshold $\kappa$ (a constant). Otherwise, the cost is either one or two coins. Notice that the possible IPCIs of $E_{2}$ are [0] and [1]. Suppose that under IPCI [0], one inserts a coin and then tries the coffee button. Regardless of the waiting time before pressing the button after the first coin, the request will be refused. In order to get coffee, he/she has to insert an additional coin. On the other hand, transition $\tau$ may occur under IPCI [1] after an insertion of a coin. After this first coin, there are two states $s_{1}$ and $s_{3}$ that are reachable, where the vending machine might lock its stability (i.e. commit) after waiting. If the lock occurs at $s_{1}$ ( $s_{3}$, respectively), the cost for coffee 
will be two coins (one coin, respectively). The underlying motivations of this relaxed interpretation are: 1) to avoid the concept of "quickly offer" (Langerak, 1990) an event which may deemed to be impractical in real applications, and 2) to bring a robust notion of stability (through stability locks) to unstable ESSs, which allows to bring closer our systems to the traditional sequential/deterministic systems.

Coffee and tea having been treated as resources, it is easier now to project on the practical aspects of ESSs. In practical situations, one may include several system parameters (as environment parameters) to anticipate and formulate interactions policies under various environment conditions, to establish discriminating views of the system with respect to the classes of potential users, and to enforce such views to maintain/converge towards a normal operational mode of the system or to meet certain reliability requirements. The underlying concepts of ESSs are of particular interest to client-server applications, communication systems, critical-safety systems, etc- which can be applied towards the specification and implementation of systems with improved reliability.

\section{The Elements of Our Testing Approach}

Given an ESS $E$, the problem of testing $E$ in our framework translates into the following: 1) environment set-up which consists of adjusting $E$ 's environment to satisfy a feasible IPCI based on $e n v_{p}^{E}$ and $e n v_{i p}^{E}$; and 2) derivation and application of tests using $S^{E}$ as a reference specification, which is the result of our weak characterization of ESSs. Throughout the paper, it is assumed that a test is applied under a feasible IPCI. Since the attachment of the predicates in $e n v_{i p}^{E}$ to $S^{E}$ is not known, the second aspect of the problem has been reduced to resolving the problems of test derivation and the procedural application of tests based on $\left\langle S^{E}, \emptyset, \Lambda\right\rangle$ ( $\Lambda$ denotes an empty list). For readability, such system notation will be referred to -interchangeably- as $S^{E}$ or $S$.

Our approach is conceptually based on the (intuitive) basic idea of $n$-testers (Pitt and Freestone, 1990) and a weaker- but modified- notion of traces than failure traces (Langerak, 1990) called Quasi-Refusal (QR) traces. It is qualified as such as we make only partial use of the unspecified interactions at some states. We shall extend the notion of $n$-testers to allow for the observation of deadlocks (or refusals) and continue testing afterwards, which leads to what we call $n$-QRtesters and their corresponding formal notion of implementation $Q R i m p l_{n}$ (short for $n$-QRimplementation)- for better conforming implementations that capture our intuitiveness of nondeterminism. For practical testing, we limit the deadlocks that appear in our traces to those that can discriminate between states that are nondeterministically reachable.

For testing ESSs, we advance a more relaxed and concrete version of Wait for Stability (Langerak, 1990), called Wait and Lock Stability: it could be possible to postulate a maximum response time for a given system; after the maximum time, the system is assumed to have locked its stability. In the presence of internal actions, the system may elect to take an arbitrary sequence- including the empty sequence- of internal actions based on its current IPCI, but the stability is locked afterwards. We assume fairness between such arbitrary choices so that one can get all possible observations under a given feasible IPCI. Our detection of deadlocks is as feasible as Langerak's because the only 
element of difference is that we do not assume that waiting leads implicitly to a stable state. For this end, suppose that the "locked stability" were waved off and that the system has reached stability in a given state after the postulated maximum time; if an internal event occurs afterwards because stability is not locked this would be a violation of the maximum response time for stability, and thus, it is also a violation of Langerak's postulate. Most importantly, locked stability can be easily realizable in practice since the action lock stability can be implemented as commit to a state. The consequences of this postulate will be apparent in Section 5. Moreover, our testing postulate is of practical significance in that testing unstable systems will translate into testing a stable system with unknown stability locks, which gives rise to the notion of stable testers. Throughout, the use of the expression "wait(ing) for stability" should be understood in the context of our postulate, i.e. "wait(ing) for stability to be locked". Also, any subsequent reference to an LTS or one of its variant forms must be taken in the context of being a component to an ESS. Finally, it is assumed that there are no internal actions that result in timeouts for clarity of exposition. We defer the treatment of such events to a later stage.

\section{Finite Tree Labelled Transition Systems}

The testing process is finite, and so must be the test sequence interactions. Accordingly, we reduce the number of executed events in the LTS component of an ESS to a certain maximum bound $n$.

Definition 2 Let $S$ be an LTS. A state $s$ in TLTS $(S)$ is said to be further-expandable if $\exists \mu \in \sum \cup\{\tau\}$ such that $s \stackrel{\mu}{\longrightarrow}$ in $\operatorname{TLTS}(S)$.

Definition 3 Given an LTS $S$ and an arbitrary number $n$, we define $T L T S_{n}(S)$ as the finite tree obtained from $T L T S(S)$ by truncating every path $s_{0}, s_{1}, \ldots, s_{k}, s_{k+1} \ldots$ of $\operatorname{TLTS}(S)$ to path $s_{0}, s_{1}, \ldots, s_{k}$ such that the following conditions hold:

1. $s_{0} \stackrel{\sigma}{\Longrightarrow} s_{k} \wedge \sigma \in \Sigma^{*} \Longrightarrow|\sigma| \leq n$,

2. $|\sigma|<n \Longrightarrow s_{k}$ is not further expandable in $T L T S(S)$, and

3. $s_{k}$ is further expandable in $\operatorname{TLTS}(S) \Longrightarrow$ Event $\left(\right.$ parent $\left.\left(s_{k}\right) \longrightarrow s_{k}\right) \in \sum$.

The first condition indicates that all traces of $T L T S_{n}(S)$ are of length less than or equal to $n$ (action $\tau$ is considered of length 0 ). The second condition indicates that such traces are of length strictly less than $n$ only if the ending states of the corresponding paths are not further expandable. The last condition of the definition ensures the unicity of the paths in $T L T S_{n}(S)$. Clearly, one can always add chains of internal events to the leaves of a $T L T S_{n}()$ without affecting the satisfiability of the first two conditions. Such ambiguity is avoided in our definition. Note these chains of internal events will be considered in $T L T S_{n+1}()$.

We do not use the distance from the root for the derivation of finite trees on the basis that there are varied degrees of nondeterminism induced by internal events in specifications, and that the assessment of conformance (testing confidence) implied by $n-Q R$ testers should not be dependent on such factor. The construction process of $T L T S_{n}()$ is finite 
under the assumption of no divergences in specifications. For a given LTS $S$ that describes a finite process, we note that there exists $k$ such that $\operatorname{TLT} S_{k}(S)=T L T S(S)$.

\section{Treatment of Internal Events by Fragmentation}

In this section, we propose a new technique for the treatment of internal events based on the fragmentation of initial LTS specifications. We first provide its primitive form and then its closure process. The primitive form is viable in that the validity of transformations with respect to our postulate becomes intuitive. The goal is to generate stable weaklyinitial labelled transition systems that simulate (step by step) the initial LTS specification and vise-versa, under our testing postulate.

Recall that we have used $S=\left(Q, \sum, \Delta, s_{0}\right)$ to denote an (initial) LTS $S$ with initial state $s_{0}$. Similarly, we write $S=\left(Q, \sum, \Delta, I Q\right)$, where $I Q \subseteq Q$, to denote a weaklyinitial LTS $S$ with $I Q$ prescribed as the set of all possible initial states of $S$. This qualification of LTSs by initial and weakly-initial has been borrowed from Starke (1972), used in the context of nondeterministic automata. The notions of stability/unstability of a state or the LTS as a whole are the same for both models. A weakly-initial LTS is thus stable if all its states are stable. To accommodate for such weakly-initial LTS, we define $\operatorname{Tr}(S)=\left\{\sigma \in \Sigma^{*} \mid s_{i} \stackrel{\sigma}{\Longrightarrow} \wedge s_{i} \in I Q\right\}$ and $S$ after $\sigma=\left\{s \mid s_{i} \stackrel{\sigma}{\Longrightarrow} \wedge \wedge s_{i} \in I Q\right\}$ with $\sigma \in \Sigma^{*}$.

\subsection{Primitive Fragmentation Process}

Contrary to other mechanisms for the removal of internal transitions (Cavalli, Kim and Maigron, 1993) (Drira,1994), we propose a fragmentation based technique of initial LTS specifications. In this section, we focus on the primitive form of this process and some of its properties.

For weakly-initial LTSs, we define the forest counterpart of tree LTS representation: a forest LTS (FLTS) is a collection of components, where each is a TLTS. A partial ordering by level of a set of states $Q$ of an FLTS is a sequence $L_{0} L_{1} \ldots L_{k}$, where $L_{i}(0 \leq i \leq k)$ is a set of the states at level $i$ in the FLTS (notice the roots are considered to be of level 0 ).

Definition 4 Let $S$ be an unstable FLTS with a set of states $Q$. Let $d^{+}(s)$ denote the number of outgoing edges for $s \in Q$. The primitive form of the fragmentation process of $S$ is described by the following sequence of actions:

1. Partially order by level the set $Q$ of FLTS $S$;

2. Select the first unstable state $s$ in the above partial order;

3. Perform one of the following actions if its applicability conditions are satisfied:

- If parent $(s) \neq$ nil and $d^{+}(s)>1$, apply the operation described in Figure 4.

- If parent $(s) \neq$ nil and $d^{+}(s)=1$, apply the operation described in Figure 5 .

- if parent $(s)=$ nil and $d^{+}(s)>1$, apply the operation described in Figure 6 .

- if parent $(s)=$ nil and $d^{+}(s)=1$, apply the operation described in Figure 7. 


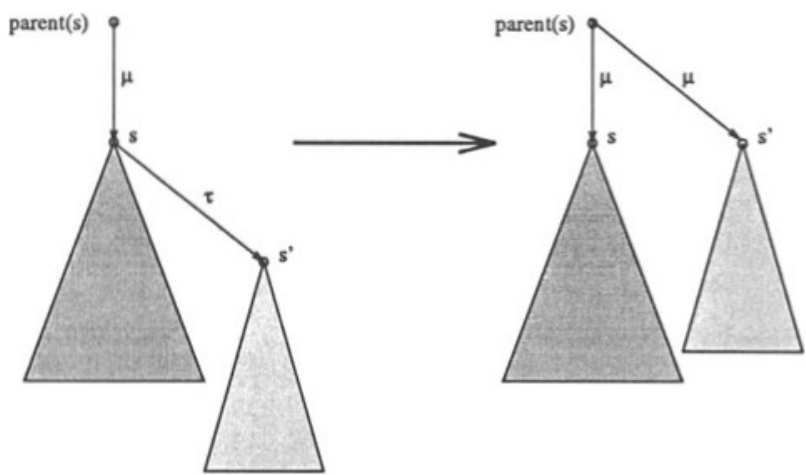

Figure 4: Primitive Form of Fragmentation: parent $(s) \neq n i l$ and $d^{+}(s)>1 ; \mu \in \sum U\{\tau\}$.

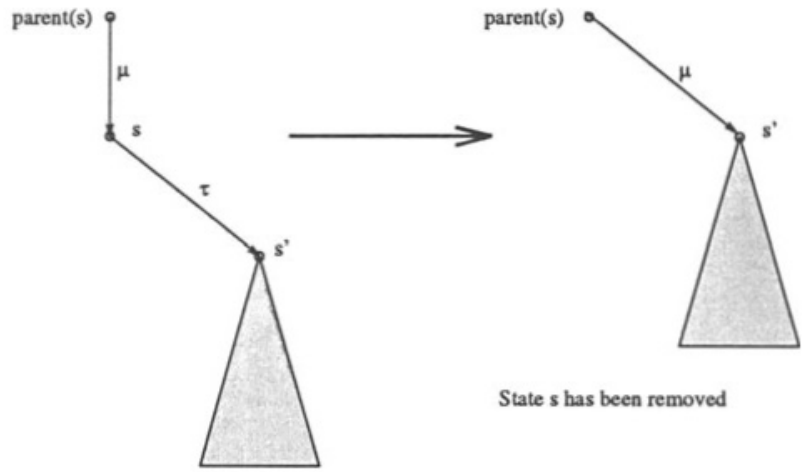

Figure 5: Primitive Form of Fragmentation: parent $(s) \neq n i l$ and $d^{+}(s)=1 ; \mu \in \sum \cup\{\tau\}$.

Dark tinted triangles in Figures 4 and 6 denote non-empty subtrees. Figure 4 depicts the fragmentation process that occurs at an unstable state $s$ that has a parent. The form of the FLTS at $s$ before fragmentation is straightforward since $s$ is unstable and $d^{+}(s) \geq 2$; if there are two or more internal transitions at $s$ then arbitrarily choose one. Similar reasoning applies to the other cases. In Figure 4, the removal of transition $s \stackrel{\tau}{\longrightarrow} s^{\prime}$ is coupled with the addition of transition parent $(s) \stackrel{\mu}{\longrightarrow} s^{\prime}$ with $\mu$ being the label of the transition from parent $(s)$ to $s$. The subtree rooted at $s^{\prime}$ remains unchanged. The intuition captured by this transformation is that if the flow of execution has reached $s$ by some trace then it is also possible that this flow of execution may have reached state $s^{\prime}$ (which is different from $s$ ). Moreover, if stability (after waiting) might have been locked at state $s$ then the internal event would not happen after this lock (otherwise, it is going to be a violation of the maximum response time). This information would be lost if states $s$ and $s^{\prime}$ were collapsed.

Figure 5 describes the degenerate case of Figure 4 when the subtree $T$ (the dark tinted triangle) is empty. In such case, we have similar basic steps coupled with the removal of 


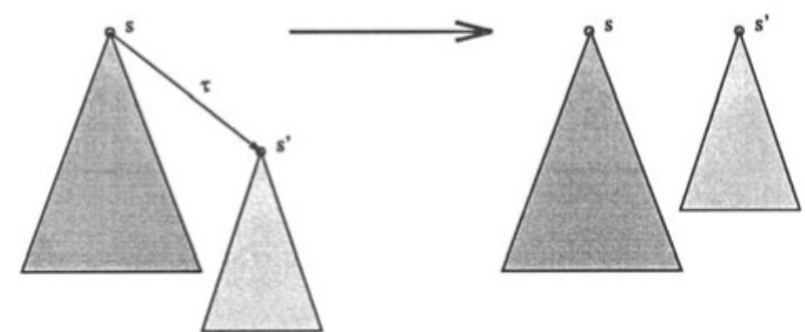

Figure 6: Primitive Form of Fragmentation: parent $(s)=n i l$ and $d^{+}(s)>1 ; \mu \in \sum \cup\{\tau\}$.
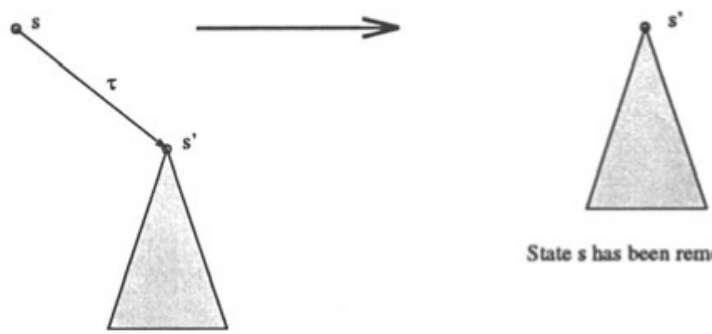

State $s$ has been removed

Figure 7: Primitive Form of Fragmentation: parent $(s)=$ nil and $d^{+}(s)=1 ; \mu \in \sum \cup\{\tau\}$.

state $s$ (notice that transition parent $(s) \stackrel{\mu}{\longrightarrow} s$ is deleted as the result of removal of $s$ ). The intuition is best described by the notion of "waiting for stability" which is part of "wait and lock stability". Under the conditions of Figure 5, state $s$ is a transient state and if we do wait for stability the flow of execution must reach state $s^{\prime}$, otherwise the lock of stability at $s$ would result in a "deadlocked" system (i.e. no subsequent progress).

Figure 6 describes an intuition similar to that of Figure 4 when state $s$ is a root. Upon the start of the system, it is possible that the system may reside in state $s^{\prime}$. However, Figure 7 is a degenerate case of Figure 6 in which the root is a transient state and if we do wait for stability the system may reside in state $s^{\prime}$.

Given an FLTS $S$, let $f p$ be an algorithm such that $f p(S)=S$ if $S$ is stable, otherwise $f p(S)$ is the result of the application of the primitive form of the fragmentation process described by Definition 4 . Hence, we have

Lemma 1 Let $S$ be an FLTS. $f p(S)$ is an FLTS.

Lemma 2 Let $S$ be an unstable FLTS. $N_{\tau}(f p(S))=N_{\tau}(S)-1$.

Proof: For any of the operations in Figures 5 to 7, the lemma is trivial. For the operation described by Figure 4, it is sufficient to observe that the number of internal transitions decreases by one in $f p(S)$ iff $\mu \neq \tau$. By partial ordering of the set of states, it follows that $\mu \neq \tau$. Thus, $N_{\tau}(f p(S))=N_{\tau}(S)-1$ in all cases. 


\subsection{Fragmentation Closure Process}

For some given LTS $S$, let $T L T S_{n}(S)=\left(Q, \sum, \Delta, s_{0}\right) . T L T S_{n}(S)$ is an initial finite LTS: it is a particular case of FLTSs. By virtue of Lemma 1 , we can recursively define $f p^{[k]}\left(T L T S_{n}(S)\right)$ for a nonnegative $k$ as follows: $f p^{[0]}\left(T L T S_{n}(S)\right)=T L T S_{n}(S)$ and $f p^{[k]}\left(T L T S_{n}(S)\right)=f p^{[k-1]}\left(f p\left(T L T S_{n}(S)\right)\right)$ when $k>0$.

The fragmentation closure process is simply $f p^{*}\left(T L T S_{n}(S)\right)=f p^{[\infty]}\left(T L T S_{n}(S)\right)$. In the remainder of this paper, we write $f p^{*}\left(T L T S_{n}(S)\right)=\left(Q^{\prime}, \sum, \Delta^{\prime}, I Q^{\prime}\right)$ where $Q^{\prime}$ is the set of states and $I Q^{\prime}$ the set of initial states of $f p^{*}\left(T L T S_{n}(S)\right)$.

Lemma $3 f p^{*}\left(T L T S_{n}(S)\right)=f p^{[k]}\left(T L T S_{n}(S)\right)$, where $k$ is $N_{\tau}\left(T L T S_{n}(S)\right)$.

Proof: It is trivial by mathematical induction on $k$ : use Lemma 2.

Lemma $4 f p^{*}\left(T L T S_{n}(S)\right)$ is a stable FLTS.

Proof: $f p^{*}\left(T L T S_{n}(t)\right)$ is an FLTS by Lemma 1 and stable by definition of $f p^{*}$.

We now refine the notion of unstability of states so that we can account for the states where the system might lock its stability.

Definition 5 Let $s$ be an unstable state. State $s$ is said to be definitely-unstable (dunstable) if all its immediate transitions are internal transitions. A state $s$ that is unstable but not d-unstable is said to be potentially-unstable (p-unstable).

We note that p-unstable states (not d-unstable states) are legitimate states where a system might lock its stability after waiting for stability; this is not allowed under Langerak's "wait for stability". Some properties of $f p^{*}$ are given below; see Ghriga (1995) for details.

Lemma 5 The following properties hold:

1. $Q^{\prime}=Q \backslash U_{d}\left(T L T S_{n}(S)\right)$, where $U_{d}\left(T L T S_{n}(S)\right)$ is the set of d-unstable states in $T L T S_{n}(S)$.

2. $I Q^{\prime}=\left(T L T S_{n}(S)\right.$ after $\left.\epsilon\right) \cap Q^{\prime}$.

3. $\operatorname{Tr}\left(f p^{*}\left(T L T S_{n}(S)\right)\right)=\operatorname{Tr}\left(T L T S_{n}(S)\right)$.

Theorem $1 f p^{*}\left(T L T S_{n}(S)\right)$ is unique.

Proof: Let $d_{\max }$ be the number of branches in the longest path in $T L T S_{n}(t)$. $f p^{*}$ works in stages due to the partial ordering of states. Hence, we can write $f p^{*}=f p^{[k]}=f p^{\left[k_{l}\right]}$ 。 $f p^{\left[k_{l-1}\right]} \circ \ldots \circ \circ f p^{\left[k_{0}\right]}$ where $k_{0}+\ldots+k_{l-1}+k_{l}=k\left(=N_{\tau}\left(T L T S_{n}(S)\right)\right)$ and $f p^{\left[k_{i}\right]}$ represents $k_{i}$ applications that are needed to make level $i$ stable (that is, all states of level $i$ stable) given that levels $i-1$ down to 0 are stable. Let $f p_{i}$ denote $f p^{\left[k_{i}\right]} \circ f p^{\left[k_{i-1}\right]} \ldots \circ f p^{\left[k_{0}\right]}$. Now, one can easily show- by induction on $i$ - that $\forall i,\left(0 \leq i \leq l<d_{\max }\right): f p_{i}\left(T L T S_{n}(S)\right)$ is unique.

Given a TLTS or an FTLTS, a path is said to be complete if the originating state (or node) is a root and the ending state is a leaf. 
Theorem 2 Given an LTS $S$, let $T L T S_{n}(S)=\left(Q, \Sigma, \Delta, s_{0}\right)$. (1) $f p^{*}\left(T L T S_{n}(S)\right)=$ $\left(Q^{\prime}, \sum, \Delta^{\prime}, I Q^{\prime}\right)$ is a unique stable weakly-initial LTS with $Q^{\prime}=Q \backslash U_{d}\left(T L T S_{n}(S)\right)$ and $I Q^{\prime}=\left(T L T S_{n}(S)\right.$ after $\left.\epsilon\right) \cap Q^{\prime}$ that is trace-equivalent to $T L T S_{n}(S)$; and (2) there is a bijection between the set $\Pi_{1}$ of complete paths in $T L T S_{n}(S)$ and the set $\Pi_{2}$ of complete paths in $f p^{*}\left(T L T S_{n}(S)\right)$.

Proof: (1) See Lemmas 4, 5, and Theorem 1. (2) Consider the following characteristic behavior function $\chi$ on paths such that $\chi(p)=\chi\left(t_{1}\right) \chi\left(t_{2}\right) \ldots \chi\left(t_{k}\right)$, where $p=t_{1} t_{2} \ldots t_{k}$ (a sequence of transitions) and $\chi\left(t_{i}\right)=\left(s_{i}, \mu_{i+1}\right)$ iff $\left(t_{i}=\left(s_{i}, \mu_{i+1}, s_{i+1}\right)\right.$ and $\left.\mu_{i+1} \neq \tau\right)$; otherwise $\chi\left(t_{i}\right)=\epsilon$. Now, it is trivial to see that there is a bijection $f$ from $\Pi_{1}$ to $\Pi_{2}$ such that $f(p)=p^{\prime}$ iff $\chi(p)=\chi\left(p^{\prime}\right)$. (Notice that this bijection is maintained after each individual application of a primitive fragmentation operation throughout the steps taken to reach $\left.f p^{*}\right)$.

Accordingly, $T \operatorname{LTS}_{n}(S)$ and its $f p$ closure simulate one another (in a step-by-step fashion) under our testing postulate. It follows that

Corollary $1 \forall \sigma \in \Sigma^{*}: f p^{*}\left(T L T S_{n}(S)\right)$ after $\sigma=\left(T L T S_{n}(S)\right.$ after $\left.\sigma\right) \cap Q^{\prime}$.

\section{Derivation of Conformance Testers}

In this section, we propose an algorithmic characterization for the construction of stable testers and provide their induced notion of implementation with an inherent procedural requirement for testers.

Definition 6 Let $S=\left(Q, \sum, \Delta, I Q\right)$ be a stable FLTS with a set of initial states $I Q \subseteq Q$. We define $\xi(S)$ as an FLTS that satisfies the following conditions:

1. Each state (or node) $s$ in the FLTS $S$ has an extra label "RS(s)" which is a refusal set at $s$ with $R S(s) \subseteq \sum$.

2. For each state $s \in Q, \mu \in R S(s)$ iff there exists $s^{\prime} \in Q$ and a trace $\sigma \in \operatorname{Tr}(S)$ such that $s, s^{\prime} \in(S$ after $\sigma)$ and $s \stackrel{\mu}{\longleftrightarrow}$ but $s^{\prime} \stackrel{\mu}{\longrightarrow}$.

The elements of a refusal set are the deadlocks that may be observed during testing. Transformation $\xi$ extends a stable FLTS with only the deadlocks that can discriminate a state from others that are simultaneously reachable. The refusal sets help check the nondeterministic structure of implementations. Clearly, $\xi(S)$ is unique for any stable FLTS.

Definition 7 Given an LTS $S, \xi \circ f p^{*}\left(T L T S_{n}(S)\right)$ is said to be $n$-QRtester of $S$.

Such $n$-QRtesters are unique for any LTS; they will be run in parallel with implementation processes under test and observe the traces with refusals. Testing an LTS implementation process of an ESS translates into testing in the presence of the unknown stability locks taken by the implementation process. This is due to i) the unknown nature of the nondeterministic transitions that are not internal that are taken under a feasible 


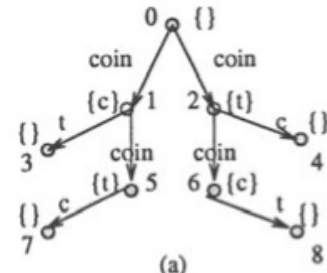

(a)

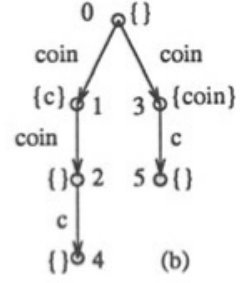

(b)

Figure 8: Examples of $n$-QRtesters. (a) 3-QRtester of $S^{E_{1}}$. (b) 3-QRtester of $S^{E_{2}}$.

IPCI, and ii) the unknown arbitrary sequences of internal transitions taken by an implementation process. Under our postulate, the problem of dealing with these two latter items becomes equivalent to finding out what stability locks (after waiting for stability) the implementation has chosen. Since the locks are unknown, we define the notion of (finite) quasi-refusal traces of an LTS component, whether stable or unstable. These traces will allow us to discriminate between the states that holds the stability locks.

Definition 8 Let $S$ be a LTS. Let $\xi \circ f p^{*}\left(T L T S_{n}(S)\right)=\left(Q^{\prime}, \sum, \Delta^{\prime}, I Q^{\prime}\right)$. Let $\varrho \in$ $\operatorname{Tr}\left(T L T S_{n}(S)\right)$. This means that $\exists s_{1}, s_{2}, \ldots, s_{k} \in Q^{\prime}: w_{0} \stackrel{a_{1}}{\longrightarrow} s_{1} \stackrel{a_{2}}{\longrightarrow} s_{2} \ldots \stackrel{a_{k}}{\longrightarrow} s_{k}$ in $\xi \circ f p^{*}\left(T L T S_{n}(S)\right)$ where $w_{0} \in I Q^{\prime}$ and $\varrho=a_{1} a_{2} \ldots a_{k}$ with $a_{i} \in \sum, 1 \leq i \leq k$.

A trace $\rho=A_{0} a_{1} A_{1} a_{2} A_{2} \ldots a_{k} A_{k}$ obtained from $\rho$ such that $A_{i} \subseteq R S\left(s_{i}\right)$ is called a quasi-refusal trace of $T L T S_{n}(S)$. The set of all quasi-refusal traces of $T L T S_{n}(S)$ will be denoted by $Q R T r_{n}(S)$.

These quasi-refusal traces allow us also to test whether the implementation exhibits the nondeterministic structure of the specification or not. This effect results from capturing the refusals (observed during a run of an $n$-QRtester with an implementation) that help us gain discrimination between the states that hold the stability locks. For a formal treatment of this issue and detectability analysis of structural abnormalities in implementations, see Ghriga (1995). As an example, Figure 8 depicts the 3-QRtester of $S^{E_{1}}$ and the 3QRtester of $S^{E_{2}}$ that have been introduced in Section 2. Notice that $T L T S_{3}\left(S^{E_{1}}\right)=S^{E_{1}}$ and the same holds for $S^{E_{2}}$. To find the quasi-refusal traces of $S^{E_{1}}$, one has to augment its traces at any given state by sets of refusals which are subsets of their corresponding $R S()$. For example, the quasi-refusal traces associated with the trace coin coin $c$ in $S^{E_{1}}$ are as follows. Notice this trace corresponds to the traversal of $s_{0} \stackrel{\text { coin }}{\longrightarrow} s_{1} \stackrel{\text { coin }}{\longrightarrow} s_{5} \stackrel{c}{\longrightarrow} s_{7}$ in $\xi\left(S^{E_{1}}\right)$; notice that $S^{E_{1}}$ is initially stable. We can construct a quasi-refusal trace by inserting a subset of $R S\left(s_{0}\right)$ at $s_{0}$, a subset of $R S\left(s_{1}\right)$ at $s_{1}$, and so on. For example, \{\} $\operatorname{coin}\{c\} \operatorname{coin}\{\} c\{\}$ is a quasi-refusal of $S^{E_{1}}$ : the \{\} $\mathrm{s}$ (or their equivalent representation D) are important and denote the fact that the system has to wait for stability. The same semantics is attached to non-empty refusal sets. Semantically (and by Definition 8 also), the trace \{\} $\operatorname{coin}\{c\}$ coin $c\{\}$ is not a quasi-refusal trace of $S^{E_{1}}$. We would like to point out that the 3-QRtester of $S^{E_{2}}$ has a different structure than its initial specification because of $f p^{*}: f p$ has been applied once to remove the initial internal transition.

Based on this notion of quasi-refusal traces under wait and lock stability, the sets (whether empty or not) are indications that our $n$-QRtesters behave in a step by step fashion and before each step (i.e. before offering an event) the tester has to wait for 
stability, after which the stability of the system is assumed to be locked and then offer an event. Notice that the empty set at the end of our sequences should not be removed as there are particular instances where these sets are not empty. The semantics associated with these empty sets are consistent with Langerak's. We do not allow for the possibility of replacing $R S()$ by an $\epsilon$ (generally speaking, "quickly offer" an event according to Langerak's TLOTOS framework) as a result of our testing assumptions.

We also would like to point out that the behavior of an $n$-QRtester can be improved. Suppose that all nodes of $T L T S_{n}(S)$ have been labelled by "d-unstable", "p-unstable" and "stable" and all transformations are made to preserve such labelling. After the required transformations, the $n$-QRtester has stable states only labelled by "p-unstable" and "stable". If the current set of states at any stage of testing contains only states labelled "stable", then the $n$-QRtester can safely override the waiting for stability of our postulate.

Before we define our notion of implementation (or, notion of conformance), it is important to note that a successful execution of a quasi-refusal trace \{\}$a_{1}\{\} a_{2}\{\} \ldots\{\} a_{k}\{\}$ by an $n$-QRtester establishes that the implementation exhibits the trace $a_{1} a_{2} \ldots a_{k}$. Similarly, a successful execution of a quasi-refusal trace $A_{0} a_{1} A_{1} a_{2} A_{2} \ldots a_{k} A_{k}$ establishes that the implementation exhibits the trace that is obtained from $A_{0} a_{1} A_{1} a_{2} A_{2} \ldots a_{k} A_{k}$ by removing the empty refusal sets.

Before we define the notion of $n$-QRimplementation, we need to define the concatenation of traces with sets of refusals. Let $\sigma=\sigma_{1} a_{1} A_{1}$ and $\sigma^{\prime}=A_{2} a_{2} \sigma_{2}^{\prime}$ where $a_{1}, a_{2} \in \sum$ and $A_{1}, A_{2} \subseteq \sum$. The concatenation of $\sigma$ and $\sigma^{\prime}$ is $\sigma \sigma^{\prime}=\sigma_{1} a_{1} A a_{2} \sigma_{2}^{\prime}$ where $A=A_{1} \cup A_{2}$.

Definition 9 Let $S$ be a LTS and I its implementation process. Implementation I is said to be an $n$-QRimplementation of $S$, written I QRimpl $S$, if

1. $Q R \operatorname{Tr}_{n}(S) \subseteq \operatorname{Tr}(I)$, and

2. $\forall \sigma \mu\{\} \in Q R T r_{n}(S):(I$ after $\sigma)$ ref $\{\mu\} \Longrightarrow \sigma\{\mu\} \in Q R T r_{n}(S)$.

The first condition expresses trace inclusion: all quasi-refusal traces $Q R \operatorname{Tr}_{n}(S)$ must be exhibited by the implementation. The second condition, however, states that the implementation should not deadlock more often than $\xi \circ f p^{*}\left(T L T S_{n}(S)\right)$ when placed in the $n$-QRtester environment whose traces are limited to $Q R T r_{n}(S)$. In other words, all refusals that occur during testing for the traces of the $n$-QRtester must be contained within its traces. This same condition makes explicit the sequentiality aspect of the application of tests. It places a procedural requirement on any tester that checks for $Q R i m p l_{n}$. By complying to this requirement, the procedural step-by-step behavior of a $n$-QRtester that is run in parallel with an implementation under test is formally described. Let $\sigma \mu\{\} \in Q R T r_{n}(S)$. If there were a successful interaction with $\sigma$ then the tester would offer $\mu$ and wait for observation; if this $\mu$ is refused by the implementation (formally, (I after $\sigma$ ) ref $\{\mu\}$ ) then this refusal must be within the behavior of the tester (formally, $\left.\sigma\{\mu\} \in Q R T r_{n}(S)\right)$. We now would like to show how one would test for a set of refusals sequentially. The trace $\sigma$ indicated above can be written as $\sigma^{\prime} A$ where $A$ is a set of refusals which may eventually be empty. By definition of the concatenation operation, $\sigma\{\mu\}$ is basically $\sigma^{\prime} A^{\prime}$ with $A^{\prime}=A \cup\{\mu\}$; we use such process to gather the set of refusals. The validity of such operation follows directly from the concept of "locked stability": once 
stability is locked at a stable state or a p-unstable state, a process progresses (or moves from its current state) only by one of its specified interactions. In the context of stable states, this coincides with the usual argument in failure trace semantics.

Given an ESS $E=<S^{E}, e n v_{p}^{E}, e n v_{i p}^{E}>$, our notions of $n$-QRtesters and $Q R i m p l_{n}$ allow us to check for conformance and define a notion of implementation using a reference ESS $\left\langle S^{E}, \emptyset, \Lambda\right\rangle$. Testing for our desired containment of the intuitive behavior of $E$ in an implementation under test would be impossible without consideration of the environment set-up for test runs. Several runs under a feasible IPCI will be scheduled to get all possible observations under such environment conditions. This same approach for gathering observations (and applying them towards our desired coverage as indicated by $Q \operatorname{Rimpl}_{n}$ ) will be followed for all feasible IPCIs.

\section{Related Work}

We relate our induced notion of conformance (or implementation) to conf, ext and examine the correlation between our traces and failure traces (Langerak, 1990). For comparative analysis, we subject testing for $\underline{\operatorname{conf}}$ and $\underline{e x t}$ to our testing postulate to mean that the set of tests of these relations remain the same but the waiting and the stability locks are an integral part for testing with respect to these relations.

Theorem 3 Let $S$ be a finite LTS and I its implementation process. Let $n$ be the smallest nonnegative number such that $T \operatorname{LT} S_{n}(S)=T L T S(S)$.

$I$ QRimpl $S \Longrightarrow I$ conf $S$ (under "wait and lock stability").

Proof: Let an implementation process $I$ be such that $I Q R i m p l_{n} S$. Suppose that $I \neg$ conf $S$. It follows that $\exists \sigma \in T L T S_{n}(S), \exists A \subseteq \sum$ such that (I after $\sigma$ ) ref $A$ but $(S \overline{\text { after }} \sigma)$ ref $A$ does not hold. This means that $\forall s \in(S$ after $\sigma), \exists \mu \in A: s \stackrel{\mu}{\Longrightarrow}$. It follows that $S$ after $\sigma$ contains at least a state $s^{\prime}$ which is not d-unstable. By Corollary 1 , we have $\forall s \in\left(f p^{*}\left(T L T S_{n}(S)\right)\right.$ after $\left.\sigma\right), \exists \mu \in A: s \stackrel{\mu}{\longrightarrow}$ and $f p^{*}\left(T L T S_{n}(S)\right)$ after $\left.\sigma\right)$ is not empty (it contains at least $s^{\prime}$ ). Therefore, $\sigma^{\prime} \mu\{\} \in Q R T r_{n}(S)$ and $\sigma^{\prime}\{\mu\} \notin Q R T r_{n}(S)$ where $\sigma^{\prime}=\{\} a_{1}\{\} a_{2}\{\} \ldots\{\} a_{k}\{\}$ with $a_{1} a_{2} \ldots a_{k}=\sigma$. Since $\mu \in A,\left(I\right.$ after $\left.\sigma^{\prime}\right)$ ref $\{\mu\}$ which is a contradiction with $I$ being an $n$-QRimplementation of $S$ (as (I after $\sigma^{\prime}$ ) ref $A$ since testing $I$ for con $f$ is done under "wait and lock stability" and (I after $\left.\sigma^{\prime}\right)$ ref $A \Longrightarrow$

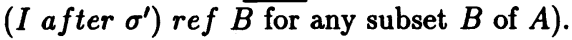

However, the converse does not hold. For example, consider the following implementation process described in LOTOS: $I=(\operatorname{coin} ;(c \rrbracket(\operatorname{coin} ; c))) \square(\operatorname{coin} ;(t[(\operatorname{coin} ; t)))$. Clearly, $I$ conf $S^{E_{1}}$ holds; see Example 1 for $S^{E_{1}}$. However, $I Q R i m p l_{3} S^{E_{1}}$ does not hold. Amongst the quasi-refusal traces of $S^{E_{1}}$ that trigger the fail verdict, we list e.g.: \{\} $\operatorname{coin}\{t\} \operatorname{coin}\{\} t\{\},\{\} \operatorname{coin}\{c\} \operatorname{coin}\{\} c\{\}$, etc.

Corollary 2 Let $S$ be an LTS and I its implementation process. Let $n$ be the smallest nonnegative number such that $T L T S_{n}(S)=T L T S(S)$.

$I$ QRimpl $n \Rightarrow I$ ext $S$ (under "wait and lock stability").

Proof: We have $\operatorname{Tr}(S) \subseteq \operatorname{Tr}(I)$ (under "wait and lock stability") since $Q R \operatorname{Tr}_{n}(S) \subseteq$ $\operatorname{Tr}(I)$. The result is then immediate by Theorem 3 . 
The converse, however, does not hold. Note that $I$ ext $S^{E_{1}}$ holds, but $I Q \operatorname{Rimpl}_{3} S^{E_{1}}$ does not hold; use the same example as above. Therefore, both conf and ext do not lead to adequate notions of conformance (or implementation) for ESSs.

Theorem 4 Let $S$ be a finite LTS process. Let $n$ be the smallest nonnegative number such that $T L T S_{n}(S)=T L T S(S)$. Let $F \operatorname{Tr}(S)$ be the set of failure traces of $S$. Let $U_{p}$ be the set of "p-unstable" states in $S . Q R T r_{n}(S) \subseteq F \operatorname{Tr}(S)$ iff $U_{p}=\emptyset$.

Proof: It is trivial by definition of failure traces (Langerak, 1990), Definition 8, Theorem $2(2)$, and the extension implied by our postulate for locking stability at "p-unstable" states.

Even though that our set of tests is not always a subset of the set of failure traces, it is significantly smaller because we make only a partial use of the unspecified interactions (i.e. deadlocks) of processes. Most importantly, the concept of stability locks has allowed us to introduce a robust notion of stability in the specification and testing of ESSs, which draws closer this class of systems to that of sequential/deterministic systems. The "stable" characteristic of our testers is a crucial attribute in gaining confidence in the observations during testing and improving the testability aspects of nondeterministic systems. Furthermore, our notion of implementation provides practical means for the conformance testing of infinite processes, which are consistent with current practices in testing.

\section{Conclusion}

Environment sensitive systems have been formally introduced. We have shown how the concept of environment sensitiveness can be used for the specification and implementation of systems with improved reliability. This particular class of systems coupled with our testing postulate Wait and Lock Stability allowed us to translate the complex problem of testing unstable systems to an equivalent problem of testing stable systems with unknown stability locks. Based on this, we have proposed new testers (that are both stable and sequential), characterized their construction, and established the correlation of their notion of implementation (with an inherent procedural requirement) to the related work in failure and failure trace semantics (when applicable). Our testers and their notion of implementation do not have an equivalent in testing theories. Most importantly, we have made an attempt to identify some of the practical aspects of nondeterminism in specifications and implementations and draw testing of LTS based implementations closer to current practices in testing; the concept of stability locks allowed us to create testing conditions similar to those of sequential and deterministic systems.

There are many directions for future research, foremost among them being to extend our testing approach to deal with data parameters and timeouts. Some other topics include the investigation of stronger notions of environment sensitive systems and development of validation tools for such systems. 


\section{Acknowledgement}

The author would like to thank Phyllis G. Frankl (Polytechnic University, NY) and the anonymous reviewers for their helpful comments and suggestions.

\section{References}

Brinksma, E. (1988) A Theory for the Derivation of Tests. Protocol Specification, Testing, and Verification VIII, (North-Holland), Elsevier Science Publishers B.V., 63-74.

Brinksma, E., Scollo, G. and Steenbergen, C (1987) LOTOS Specifications, their Implementations and their Tests. Protocol Specification, Testing, and Verification VI, (North-Holland), Elsevier Science Publishers B.V., 349-360.

Cavalli, A.R., Favreau, J.P. and Phalippou, M. (1994) Formal Methods for Conformance Testing: Results and Perspectives. Protocol Test Systems VI, (North-Holland), Elsevier Science Publishers B.V., 3-19.

Cavalli, A.R., Kim, S.U. and Maigron, P. (1993) Automated Protocol Conformance Test Generation Based on Formal Methods for LOTOS specifications. Protocol Test Systems V, (North-Holland), Elsevier Science Publishers B.V., 212-222.

Drira, K. (1994) The Refusal Graph: a Tradeoff between Verification and Test. Protocol Test Systems VI, (North-Holland), Elsevier Science Publishers B.V., 301-316.

Fujiwara, S. and Bochmann, G.V. (1992) Testing non-deterministic state machines with fault coverage. Protocol Test Systems IV, (North-Holland), Elsevier Science Publishers B.V., 267-280.

Ghriga, M. (1995) Conformance Testing of Nondeterministic Communication Systems. PhD thesis, Computer Science Department, Polytechnic University, NY.

Langerak, R. (1990) A Testing Theory for LOTOS using Deadlock Detection. Protocol Specification, Testing, and Verification IX, (North-Holland), Elsevier Science Publishers B.V., 87-98.

Petrenko, A., Bochmann, G.V. and Dssouli, R. (1994) Conformance relations and test derivations. Protocol Test Systems VI, (North-Holland), Elsevier Science Publishers B.V., 157-178.

Pitt, D.H. and Freestone, D. (1990) The derivation of Conformance Tests from LOTOS Specifications. IEEE Transactions on Software Engineering, 16, 1337-1343.

Starke, P.H. (1972) Abstract Automata. North-Holland: Elsevier Science Publishers.

Tretmans, J. (1994) A Formal Approach to Conformance Testing. Protocol Test Systems VI, (North-Holland), Elsevier Science Publishers B.V., 261-280.

\section{Bibliography}

Mohammed Ghriga received the Dipl. d'Ingénieur d'état in computer science from the University of Sciences and Technology at Algiers (USTHB), Algeria; and the M.S. and $\mathrm{Ph}$.D. degrees in computer science from Polytechnic University, Brooklyn, NY, USA.

He has been an Assistant Professor of Computer Science at Long Island University, NY, since September 1994. His current research interests include software and protocol testing, conformance testing, formal specification techniques, software verification and validation, and software testability. 